\title{
Caracterización radiométrica de una cabeza de flash para su uso en digitalización de obras de arte
}

José Manuel Pereira Uzal | conservador y restaurador de bienes culturales, editor de DigitalHeritage

URL de la contribución <www.iaph.es/revistaph/index.php/revistaph/article/view/4085>

\section{RESUMEN}

El uso del flash fotográfico como fuente de iluminación es un recurso habitual en las tareas de documentación y digitalización de obras de arte y bienes culturales; sin embargo, no está exento de sospecha por parte de algunos investigadores como factor de alteración. A lo largo de este trabajo hemos reproducido diversas experiencias de caracterización de un equipo de flash extensamente documentadas en la bibliografía de nuestro sector sin que, a priori, podamos plantear el uso racional del flash como un factor de alteración.

\section{Palabras clave}

Bienes culturales | Digitalización | Flash | Fotografía | Fuentes de iluminación | Obras de arte | Reproducción | 


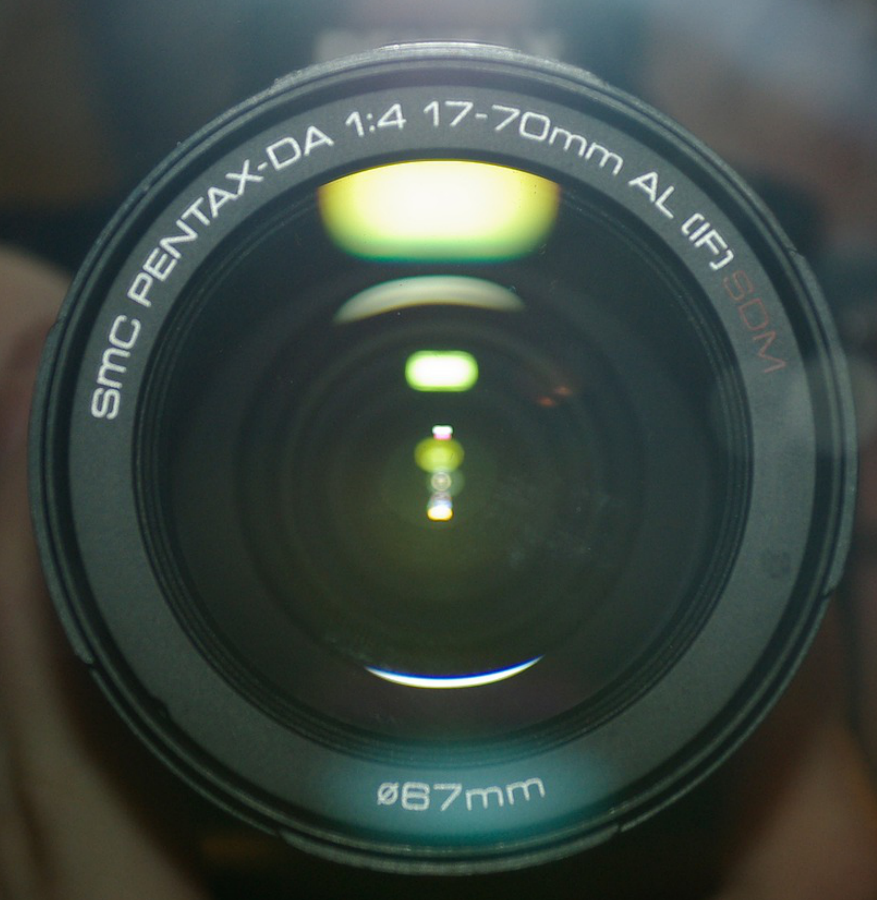

Pentax k200d | fuente Pixabay 


\section{INTRODUCCIÓN}

En la actualidad las tareas de digitalización o documentación de bienes culturales a través de equipos fotográficos o de captación de imagen son casi una actividad cotidiana. De esta forma, las fuentes de iluminación son una herramienta intrínseca a todas estas actividades.

Las fuentes o dispositivos de iluminación usados en bienes culturales pueden ser divididos en dos grandes grupos: los de luz continua, en los cuales se incluyen sistemas basados en la fluorescencia, electroluminiscencia (LED), incandescencia, HMI, etc.; y los de luz no continua, basada en destellos producidos por un arco de xenón, conocida comúnmente como luz de flash.

El uso de destellos como fuente de iluminación posee la ventaja de ofrecer una gran intensidad con tiempos de destello muy breves, lo cual permite anular cualquier trepidación en cámara, por lo que la proponen como un sistema ideal para lograr una máxima reproducción de detalle al mismo tiempo que presenta una gran eficiencia entre consumo y potencia.

En el presente artículo vamos a realizar una revisión de las estrategias de caracterización de una fuente de iluminación, así como las fuentes bibliográficas referentes a dichos dispositivos.

\section{METODOLOGÍA}

El presente estudio estará basado en la evaluación de una cabeza de flash Elinchrom RX600 sobre la cual vamos a realizar diversas mediciones, con herramientas fácilmente asequibles para cualquier profesional, a diferentes intensidades del destello y usando un modificador de luz a modo de pantalla difusora, especialmente habitual en el uso con este tipo de iluminación, con el fin de suavizar el haz de luz emitido.

Los aspectos analizados durante los ensayos se centrarán en estudiar el tiempo del destello, el contenido en radiaciones ultravioleta (UV) del mismo, así como la iluminancia y la irradiancia.

Para ello nos hemos servido de diversos instrumentos, como un espectrofotómetro i1pro de X-Rite con una sensibilidad de entre $380 \mathrm{~nm}-730 \mathrm{~nm}$, con el software de análisis BabelColor CT\&A a fin de realizar mediciones relativas a la iluminancia, CRI y Temperatura de Color Correlacionada.

Así como un microcontrolador Atmega328 montado sobre una placa Arduino, con el fin de determinar el tiempo del destello con un fototransistor de alta sensibilidad y respuesta. Y un sensor GUVA-S12SD de Roithner 


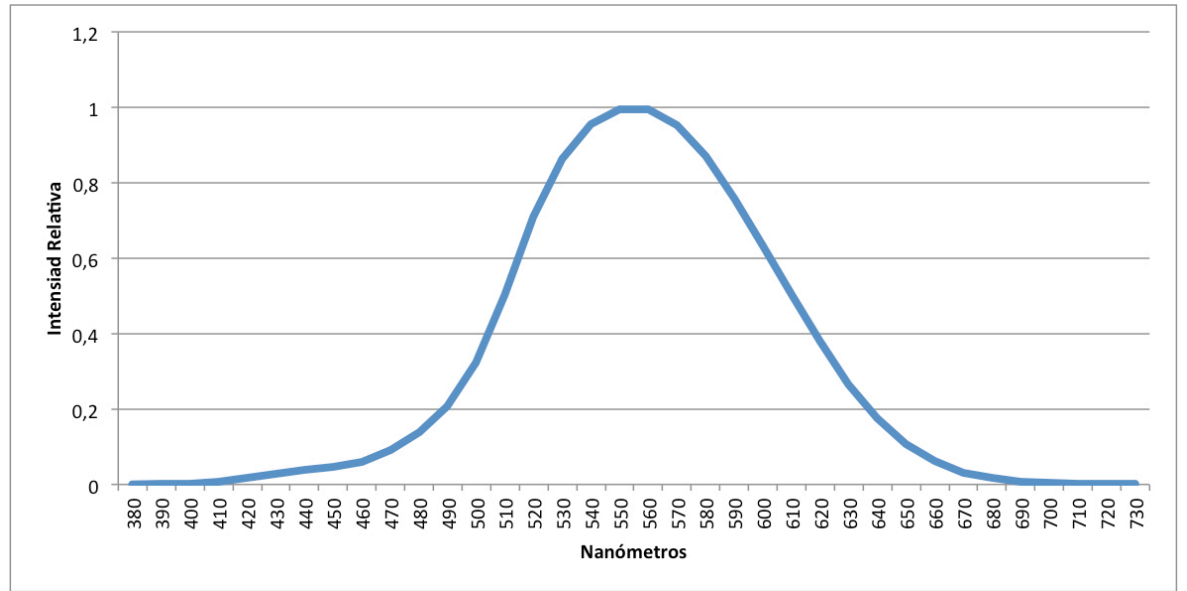

Lasertechnik con una sensibilidad entre los $240 \mathrm{~nm}$ y los $380 \mathrm{~nm}$ con el fin de estudiar la región del UV.

Las unidades utilizadas para el estudio de las diferentes características de este tipo de luz serán las siguientes:

La iluminancia descrita en Lux por segundo (Lux s), que nos informa sobre la cantidad de luz emitida por el destello en términos fotométricos; es decir, en base a la curva de respuesta fotópica a la luz del ser humano. Y en segundos, ya que al tratarse de un destello debe ser referido al tiempo tal y como lo cita el National Institute of Standards and Technology (OHNO, 2002).

Este mismo concepto de expresar la cantidad de luz en función del tiempo es igualmente utilizado para cuantificar la radiación lumínica recibida por un bien cultural; hablaríamos entonces de "exposición lumínica" descrita habitualmente por las unidades $\mathrm{H}_{v^{\prime}}$ donde:

$H_{v}=E_{v}{ }^{*} t$

Donde $E_{v}$ es la iluminancia medida en lux y $t$ el tiempo en segundos.

La problemática del estudio de la iluminancia de un destello no está exenta de dificultades, ya que la intensidad de éste raras veces es constante a lo largo del tiempo del mismo, por tanto habrá momentos del destello donde la intensidad es mayor o menor.

Por causa de este fenómeno los fabricantes de equipos de flash orientados a la fotografía hablan de las métricas T0.5 o T0.1, donde la intensidad del destello referido al tiempo del mismo se estudia cuando este ha alcanzado el 50\% de su intensidad (T0.5) o el 10\% (T0.1), de forma que los datos de
Función de luminosidad fotópica del sistema de visión del ser humano | fuente CIE 
intensidad arrojados no serán los mismos, así que un destello con un tiempo dado, para T0.1, posee más duración y por tanto emite más energía que para T0.5.

De esta forma, desde el punto de vista de la conservación, es más interesante hablar en términos de irradiancia, es decir, cantidad de energía que llega a una superficie, descrita por la métrica $\mathrm{W} / \mathrm{m}^{2}$.

Otra dificultad de cuantificar con propiedad la energía recibida por una obra en términos fotométricos es que dicha energía está siendo ponderada por la función fotópica que nos describe la sensibilidad a la luz del sistema de visión humano, de esta manera no estamos cualificando como energía que llega a una obra de arte aquellas zonas del espectro no visibles por el ojo humano.

Otro aspecto fundamental en el estudio de una fuente de iluminación es conocer su radiancia espectral, es decir la cantidad de energía emitida a lo largo de las diferentes longitudes de onda, habitualmente expresadas en $\mathrm{W}$ $\mathrm{nm}^{-1} \mathrm{~m}^{-2}$, pero que al estar hablando de un destello relativo a un periodo de tiempo se suele usar $\mathrm{J} \mathrm{nm}^{-1} \mathrm{~m}^{-2}$.

Conocer la distribución del espectro de una fuente de luz es fundamental no solo para el cálculo de la irradiancia, sino también para el de la Temperatura de Color Correlacionada (Color Correlated Temperature, CCT) o el Índice de Reproducción Cromática descrita por el CRI (Color Render Index).

Todas las mediciones han sido realizadas a 1 metro de distancia con un ángulo de haz aproximado de $65^{\circ}$ lo cual nos produce un área aproximada de $1 \mathrm{~m}^{2}$ con el fin de que 1 lux $\mathrm{m}^{2}=1$ lumen y simplificar los cálculos y apreciaciones.

\section{RESULTADOS}

A continuación se presentan algunos de los resultados obtenidos

\section{Medición del tiempo de destello}

Los tiempos de destello alcanzados fueron los siguientes:

\begin{tabular}{|lll}
\hline & Máxima potencia & Mínima potencia \\
\hline T0.5 & $1 / 446 \mathrm{~s}(0.0024 \mathrm{~s})$ & $1 / 578 \mathrm{~s}(0.0017 \mathrm{~s})$ \\
\hline T0.1 & $1 / 390 \mathrm{~s}(0.0025 \mathrm{~s})$ & $1 / 459 \mathrm{~s}(0.0021 \mathrm{~s})$ \\
\hline
\end{tabular}




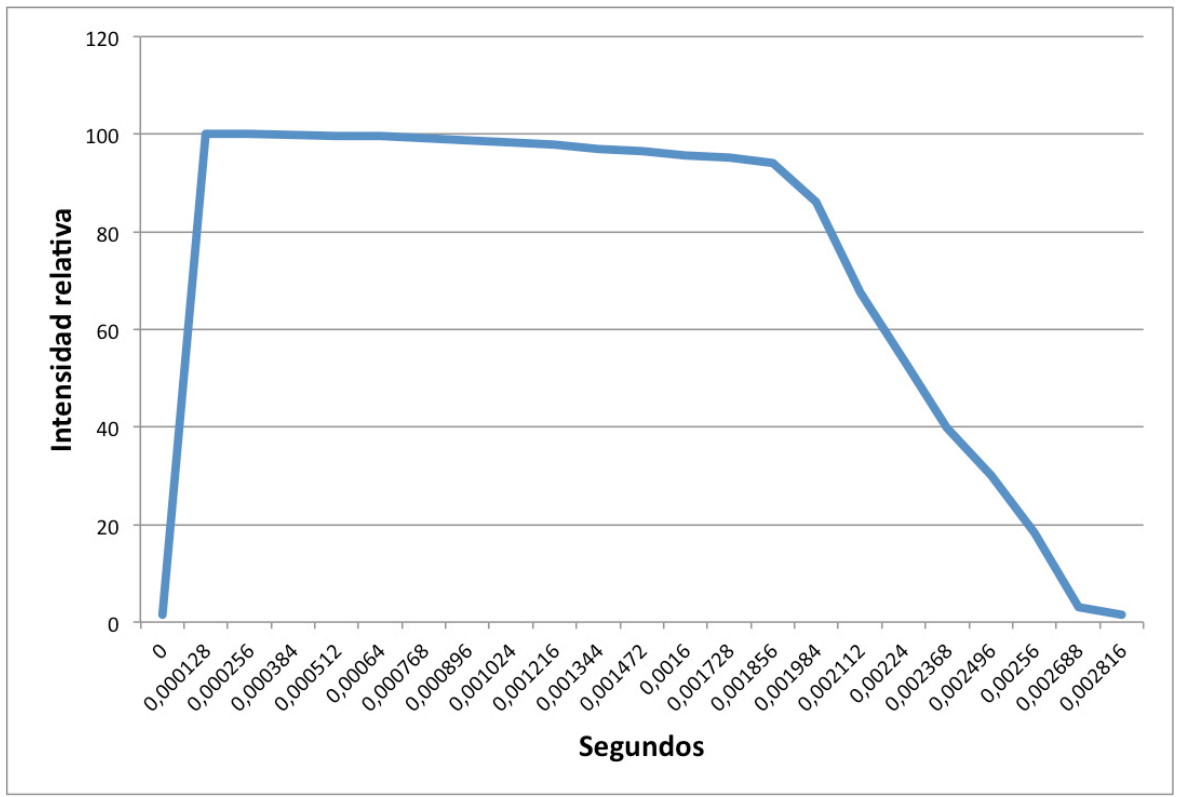

Tiempo de destello a máxima potencia

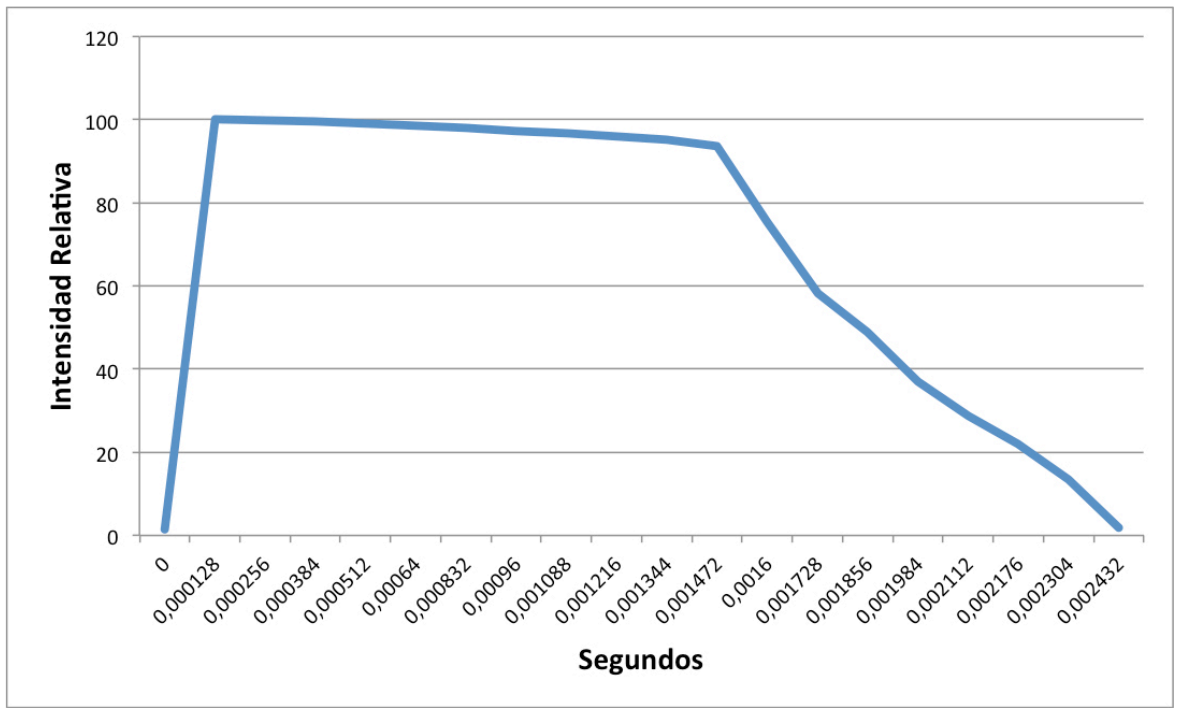

Tiempo de destello a mínima potencia

\section{Medición del contenido en ultravioleta}

\begin{tabular}{|llllll|}
\hline \multicolumn{2}{l}{ Máxima potencia } & \multicolumn{2}{l}{ Mínima potencia } & \multicolumn{2}{l|}{ Mínima potencia + Modificador } \\
\hline Promedio & Máximo & Promedio & Máximo & Promedio & Máximo \\
\hline $0.9 \mathrm{~W} / \mathrm{m}^{-2}$ & $1,16 \mathrm{~W} / \mathrm{m}^{-2}$ & $0.057 \mathrm{~W} / \mathrm{m}^{-2}$ & $0.07 \mathrm{~W} / \mathrm{m}^{-2}$ & $0.0015 \mathrm{~W} / \mathrm{m}^{-2}$ & $0.01 \mathrm{~W} / \mathrm{m}^{-2}$ \\
\hline
\end{tabular}


Relación del contenido ultravioleta y el tiempo del destello a diferentes potencias y con modificador de luz
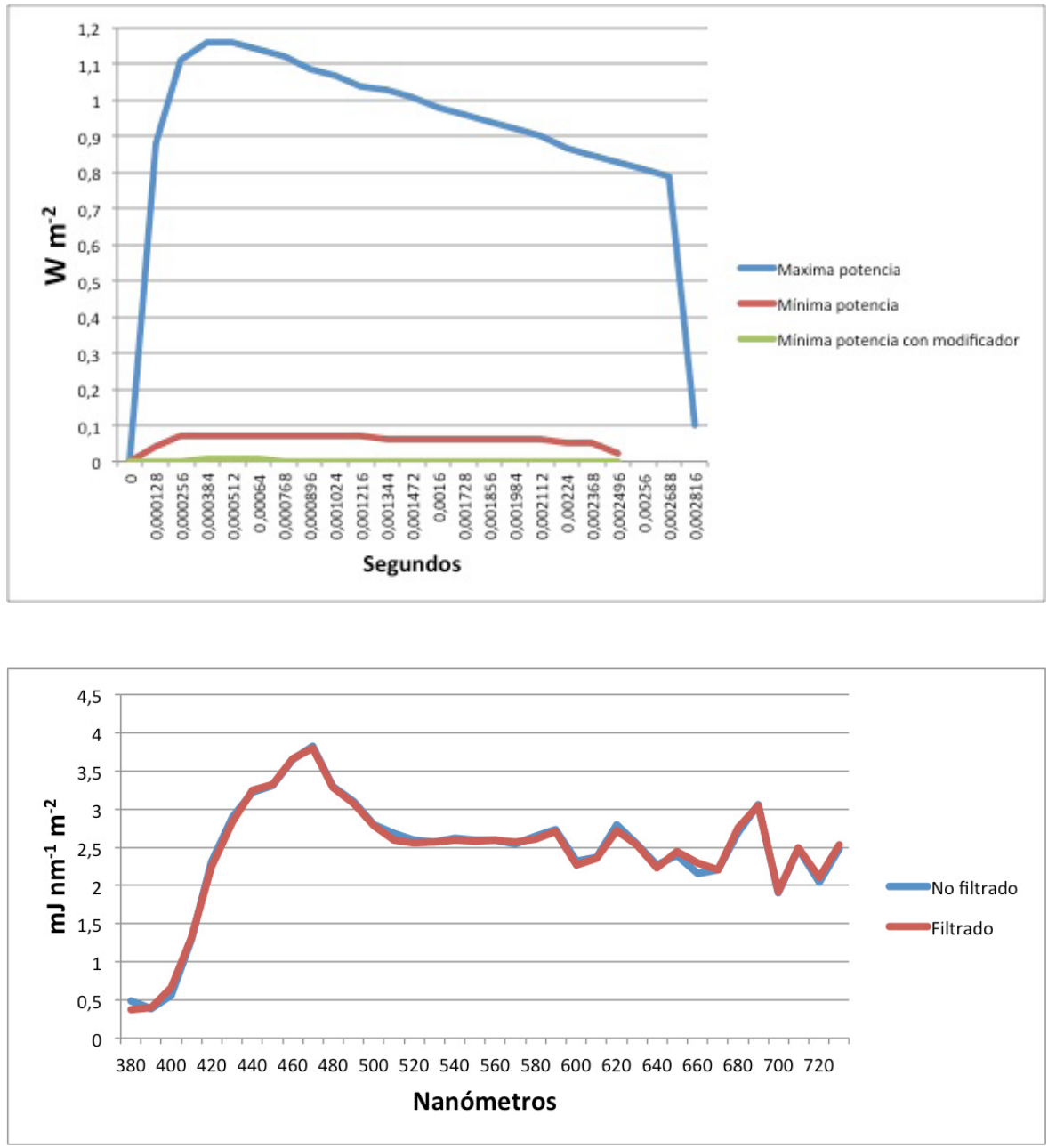

Medición de la iluminancia, CCT y CRI

\begin{tabular}{llll} 
& Iluminancia & CCT & CRI \\
\hline Sin modificador & 360 lux s & $5733 \mathrm{~K}(\mathrm{D} 57)$ & 96 \\
\hline Con modificador & 189 lux s & $6641 \mathrm{~K}(\mathrm{D} 66)$ & 93
\end{tabular}

Las mediciones aportadas son a mínima potencia, con el fin de simplificar las mediciones.

\section{Irradiancia para el espectro completo}

Además de los datos fotométricos, que pueden ser de relevancia para una estimación desde el punto de vista de la percepción del ser humano, cono- 
cer la irradiancia es mucho más relevante para evaluar la energía que llega a nuestros bienes culturales, ya que computamos el espectro completo y no solo aquella parte relativa a la sensibilidad del sistema de visión humano tal y como lo han abordado trabajos previos como el Technical Report 157 de la CIE, basado en los trabajos anteriores de Harrison (HARRISON, 1953) o los trabajos de Terry Schaeffer (SCHAEFFER, 2001).

Para ello partimos de la siguiente ecuación:

$H_{i}=\Delta \lambda \cdot F\left(\lambda_{i}\right)$

Donde $\mathrm{H}$ es la irradiancia, o densidad radiada expresada en $\mathrm{W} \mathrm{m^{-2 }}, \mathrm{F}(\lambda)$ es la irradiancia espectral expresada en $W^{-2} \mu m^{-1}$ y $\Delta \lambda$ son las longitudes de onda estudiadas.

De esta forma para un tiempo de destello de $0.0021 \mathrm{~s}$, la irradiancia de nuestro equipo sería:

\begin{tabular}{|ll|} 
& Irradiancia a baja potencia \\
\hline Sin modificador & $0.9753 \mathrm{~W} \mathrm{~m}^{-2}$ \\
\hline Con modificador & $0.9782 \mathrm{~W} \mathrm{~m}^{-2}$ \\
\hline
\end{tabular}

Si por la contra evaluamos la irradiancia exclusivamente en la fracción del UV que nuestro espectrofotómetro nos aporta entre los $380 \mathrm{~nm}$ y los $400 \mathrm{~nm}$, obtenemos los siguientes datos:

\begin{tabular}{|ll|} 
& Irradiancia a baja potencia \\
\hline Sin modificador & $0.5614 \mathrm{~W} \mathrm{~m}^{-2}$ \\
\hline Con modificador & $0.2668 \mathrm{~W} \mathrm{~m}^{-2}$ \\
\hline
\end{tabular}

Estos valores de emisión en el $\mathrm{UV}_{380-400 \mathrm{~nm}}$ se encuentran particularmente próximos a los cálculos realizados por Terry Schaeffer (SCHAEFFER, 2001) donde propone $4.5 \times 10^{-5} \mathrm{~W} \mathrm{~cm}^{-2}\left(0.45 \mathrm{~W} \mathrm{~m}^{-2}\right)$.

\section{DISCUSIÓN}

Si nos remitimos a los últimos resultados en materia de radiancia, de forma paradójica, vemos cómo para dos emisiones, con diferente iluminancia en términos de Lux s (360lux s y 160Lux s), la irradiancia es prácticamente la 
misma $0.9753 \mathrm{~W} \mathrm{~m}^{-2}$ y $0.9782 \mathrm{~W} \mathrm{~m}^{-2}$, siendo incluso menor la que no utiliza modificador de luz, a pesar de tener mayor iluminancia. Esto es debido al modificador de luz, ya que al variar la temperatura de color, ofrece más energía en las zonas de longitudes de onda más corta y hace que aumente levemente la irradiancia, aun teniendo una menor iluminancia. Esta situación nos alerta sobre las implicaciones en el uso de fuentes de iluminación más "frías", es decir, ricas en longitudes de onda más cortas frente al uso de fuentes de iluminación más "cálidas" o neutras.

De esta forma podemos adentrarnos en la transcendencia de remitirnos a la irradiancia como métrica más representativa que la propia iluminancia, ya que valoramos la energía aportada por todo el espectro estudiado y no solo aquella relativa a la percepción del ser humano.

Y, aunque podamos hablar de valores de iluminancia más o menos altos, los valores de irradiancia, sobre todo en términos relativos al contenido de radiación ultravioleta, son particularmente bajos, siempre y cuando usemos algún tipo de modificador de luz o filtro UV delante del bulbo de nuestro flash, que, habitualmente, y es la práctica de muchos fabricantes, ya ha sido montado de serie.

A nivel fotométrico nuestro equipo emite aproximadamente un flujo luminoso de 190 Lux s o 0.05 lux h, que es la intensidad necesaria para disparar a una distancia aproximada de $1 \mathrm{~m}$ a f8 y una sensibilidad de 100 ISO, ya que debe cumplirse la relación:

f-stop $=(0.003 * 100 * 190)^{1 / 2}$

f-stop $=7.5$

Donde 0.003 es una constante relativa al cálculo de la exposición referida a la ISO 2720: 1974 Photography. General purpose photographic exposure meters (photoelectric type)-Guide to product specification.

Por tanto, para una sesión aproximada de 1000 fotografías, la cantidad de energía luminosa que llega a la obra es de:

$0.05 \mathrm{Luxh} * 1000=50 \mathrm{Luxh}$

Sin embargo, aunque pueda parecer una cifra alta, si la comparamos con una emisión que proviene de una luz continua de, por ejemplo, 12 lux, tendremos que para una jornada de ocho horas de exposición al público ha emitido 96 Lux h, por lo que podemos observar que en una jornada de ocho horas de exposición se ha emitido casi el doble de radiación sobre la obra que en 1000 disparos de flash (WARE, 1994). 
Unas estimaciones similares las podemos encontrar en el trabajos de Mike Ware (1994) donde se realizan estimaciones entre los umbrales de exposición para luz continua y flash, así, por ejemplo, si un material posee un umbral de exposición de 3h a 50 lux (540.000 Lux s), podrá ser fotografiado más de 4000 veces con un flash que emita 128 lux s.

De esta forma, por ejemplo, David Saunders del National Gallery (SAUNDERS, 1995) estima una cantidad media de 0.17 lux h por cada disparo a una apertura de f8, que para una exposición anual de 600.000 lux $\mathrm{h}$ recomendado para óleo y témpera por dicha institución representan más de tres millones de disparos de flashes necesarios para alcanzar dicho umbral de tolerancia.

Garry Tompsom (2013) describe una relación similar donde le atribuye a cada disparo de flash una energía promedio de 20 lux minuto (1200 lux s; 0.3 lux h) para una apertura de f22.

Uno de los primeros estudios referente en la alteración de materiales por el efecto de la luz del flash fue el llevado a cabo por Hanlan (1970), que concluye en su trabajo con la sentencia: "No parece existir ninguna razón para ver el uso normal del flash electrónico como algo injustamente peligroso". A raíz de los resultados mostrados en el trabajo de Hanlan, el propio Garry Tompsom (2013) concluye que "podemos asegurar tranquilamente que en condiciones reales y usado con prudencia, el flash electrónico continuado es menos perjudicial que la exposición continuada bajo lámparas incandescentes. De forma similar el propio comité francés del ICOM ha llegado a unas conclusiones similares en 1969 y así lo refleja en sus recomendaciones para reproducciones de textiles "La luz más segura para la fotografía de textiles es considerada la del flash electrónico dado que las fuentes de tungsteno o lámparas de cuarzo tienen altos niveles de luz infrarroja".

En función a estos estudios previos, la National Gallery en sus recomendaciones para la fotografía y grabación profesional dentro de las salas, Guidelines for Lighting for Photography and Televisión (marzo del 2010), toma en consideración las sugeridas por Garry Tompsom y establece que para el ámbito de la fotografía profesional: "el flash electrónico está permitido y es preferido al uso de lámparas fotográficas" y éste no debe superar los 1250 lux s por cada disparo así como el uso de filtros UV específicos sobre el tubo.

En lo relativo al contenido UV, el simple uso de un modificador de luz tipo "softbox" o paraguas traslucido, en modo difusor, presenta una fuerte absorción a la región del UV motivado por la composición química de las propias fibras de poliéster del modificador. De esta forma, para el equipo estudiado, donde para una irradiancia en la región del UV de $0.0015 \mathrm{~W} \mathrm{~m}^{-2}$, y para un contenido máximo recomendado de $75 \mu \mathrm{W}$ por lumen (SCHAEFFER, 2001) 
vemos que para una iluminancia de 190 Lumen por segundo obtenemos $0.0015 \mathrm{~W} \mathrm{~m}^{-2}$, para 1 lumen tenemos $0.126 \mu \mathrm{W}$ por tanto nos encontramos muy por debajo de las recomendaciones expuestas por Schaeffer. De esta forma el propio autor estima un contenido de UV de un flash electrónico en $4.5 \times 10^{-5} \mathrm{~W} \mathrm{~cm}^{-2}$ o $4.5 \times 10^{-7} \mathrm{~J} \mathrm{~cm}^{-2}$ para un tiempo de destello de $1 / 100$.

\section{CONCLUSIONES}

Finalmente, a raíz de estos ensayos y experiencias de diversos autores a lo largo de las últimas décadas, es complejo encontrar argumentos donde el flash electrónico o flash fotográfico, correctamente filtrado en UV, supongan un daño evidente para la obra.

Según Terry Schaeffer (2001), los daños potenciales ejercidos por un flash fotográfico han podido ser relacionados con procesos bifotónicos vinculados con pulsos de luz extremadamente intensos en comparación con las emisiones de luz continua habituales. Sin embargo, según el propio Terry Schaeffer, "dichos procesos son extremadamente improbables durante el uso de flashes fotográficos" y dichos fenómenos se relacionarían, por tanto, con fuentes de luz de alta energía como los láseres.

Por tanto, no parecen justificadas las restricciones en el uso de flash sobre patrimonio cultural, ya que a raíz de nuestras observaciones, y en relación con las fuentes bibliográficas consultadas, no tenemos a día de hoy indicios suficientes para proponer el uso del flash como un factor de alteración. Por la contra, se hace conveniente fijar la atención en la iluminación a través de luces continuas de alta intensidad, ya que los tiempos de exposición de la obra se incrementan considerablemente frente al uso de luz no continua.

\section{RECOMENDACIONES}

A pesar de no tener evidencias claras del flash como factor de alteración de un bien cultural, cabe plantear un uso racional del mismo y como cualquier intervención o acción sobre un bien cultural, sus parámetros de uso, tales como potencia expresada en las unidades correspondientes, distancia, números de disparos, marca y modelo, modificadores usados, etc. debería quedar oportunamente registrado en una memoria de intervención, con el fin de dejar constancia de la radiación emitida sobre la obra para su evaluación en un tiempo futuro. 


\section{BIBLIOGRAFÍA}

- BLACKWELL, B. (2000) Light exposure to sensitive artworks during digital photography. Spectra, vol. $26, n .{ }^{\circ} 2$, 2000, pp. $24-28$

- FELLER, R. L. (1995) Accelerated aging: photochemical and thermal aspects. S. I.: Getty Publications, 1995

- GUIDELINES for Lighting for Photography and Television. National Gallery of London (2010) [en línea], The National Gallery, 2010. <https://www.nationalgallery. org.uk/media/15989/press-and-media_photography-andtelevision-lighting-guidelines.pdf> [Consulta: 06/03/2019]

- HANLAN, J. F. (1970) The effect of electronic photographic lamps on the materials of works of art. Museum news, vol. 48, n. ${ }^{0} 10,1970$, pp. 33-41

- HARRISON, L. S. (1953) Report on the deteriorating effects of modern light sources. S. I.: Metropolitan Museum of Art New York, 1953

- international COMMISSION on ILLUMINATION. TECHNICAL COMMITTEE TC 3-22 "MUSEUM LIGHTING". Control of Damage to Museum Objects by Optical Radiation. Commission internationale de l'eclairage, 2004.

- OHNO, Y. (2002) Physical measurement of flashing lights-now and then. Proc. CIE Expert Symposium on Temporal and Spatial Aspects of Light and Colour Perception and Measurements. S. I.: s.n., 2002

- SAUNDERS, D. (1995) Photographic flash: threat or nuisance? National Gallery Technical Bulletin, vol. 16, 1995, pp. 66-72

- SCHAEFFER, T. T. (2001) Effects of light on materials in collections: data on photoflash and related sources. S. I.: Getty Publications, 2001

- THOMSON, G. (2013) The museum environment. S.I.: Elsevier, 2013

- WARE, M. (1994) Mechanisms of image deterioration in early photographs. London: Science Museum and National Museum of Photography, Film \& Television, 1994 